\title{
LED and UV Light Utilizing Flavonoids and Limonoids to Investigate the Effect of Post-harvest Light Irradiation
}

\author{
Shengyu Liu ${ }^{1}$,Linping Hu ${ }^{1}$,Dong Jiang ${ }^{2}$ and Wanpeng $\mathrm{Xi}^{1,3,{ }^{1}}$ \\ 1 \\ College of Horticulture and Landscape Architecture, Southwest University, Chongqing 400716, China \\ 2
}

Citrus Research Institute, Chinese Academy of Agricultural Sciences, Chongqing 400712, China 3

Key Laboratory of Horticulture Science for Southern Mountainous Regions, Ministry of Education, Chongqing 400715, China

Author to whom correspondence should be addressed.

Received: 20 March 2019 / Accepted: 4 May 2019 / Published: 6 May 2019

\begin{abstract}
To investigate the effect of post-harvest light irradiation on the accumulation of flavonoids and limonoids, harvested Newhall navel oranges were continuously exposed to light-emitting diode (LED) and ultraviolet (UV) light irradiation for 6 days, and the composition and content of flavonoids and limonoids in the segments were determined using UPLC-qTOF-MS at 0, 6, and 15 days after harvest. In total, six polymethoxylated flavonoids (PMFs), five flavone$\mathrm{O} / \mathrm{C}$-glycosides, seven flavanone-O-glycosides, and three limonoids were identified in the segments. The accumulation of these components was altered by light irradiation. Red and blue light resulted in higher levels of PMFs during exposure periods. The accumulation of PMFs was also significantly induced after white light, UVB and UVC irradiation were removed. Red and UVC irradiation induced the accumulation of flavone and flavanone glycosides throughout the entire experimental period. Single light induced limonoid accumulation during exposure periods, but limonoid levels decreased significantly when irradiation was removed. Principal component analysis showed a clear correlation between PMFs and white light, between flavonoid glycosides and red light and UVC, and between limonoids and UVC. These results suggest that the accumulation of flavonoids and limonoids in citrus is regulated by light irradiation. White light, red light and UVC irradiation might be a good potential method for improving the nutrition and flavor quality of post-harvest citrus.
\end{abstract}

Keywords:

Newhall navel oranges; LED; UVs; PMFs; flavonoid glucosides; limonoids

\section{Introduction}

Many physical or chemical methods, such as cold storage [1], heat treatment [2], light irradiation [3], and natural antibacterial agents [4] are used to delay the loss of fruit quality after harvest. Post-harvest chemical treatments for preserving fruit are becoming less accepted by consumers due to the possible pollution or other undesired residues. Light irradiation has attracted increasing attention due to its advantages of being highly efficient and residue free as well as its ability to control decay and extend storage life. It has been reported that exposure to UVC and UVB induces large increases in resveratrol derivatives in post-harvest grapes [5]. Traditionally, UVC irradiation has been recognized as an effective sterilization method to increase the shelf-life of fruits $[6,7]$. Continuous white-blue light exposure increases the storage time of broccoli and contributes to a higher accumulation of antioxidant compounds [8]. These findings suggest that both ultraviolet and other lights have the potential to be applied for maintaining or enriching the health-promoting components of post-harvest fruits.

Previous studies have investigated the effect of red and blue LED light irradiation on $\beta$-cryptoxanthin 
accumulation in the flavedo of Satsuma mandarin (Citrus unshiu Marc.), the combination of ethylene and red LED light irradiation on carotenoid metabolism [9], and the regulation role of red and blue LED light irradiation in ascorbic acid metabolism in citrus juice sacs of Satsuma mandarin (Citrus unshiu Marc.), Valencia orange ( $C$. sinensis Osbeck), and Lisbon lemon (C. limon Burm.f.) [10]. To date, the effect of light irradiation on flavonoid metabolism in Newhall navel oranges or other citrus fruits has not yet been reported. Though similar studies were conducted in pear, apple, and mango, only total flavonoid content was determined, or single light irradiation was used. A global evaluation of multiple light sources regarding bioactive compounds in fruit is still lacking.

Flavonoids and limonoids are two major groups of health-promoting components in citrus fruits that exhibit a broad range of biological activities, such as antioxidant [11], anti-proliferative [12], anti-atherosclerotic [13], and neuroprotective effects [14]. Light treatment reportedly increases total flavonoid content in a wide range of species, including pear [15], apple [16], mango [6], and Brussel sprouts [17]. However, the majority of studies have only examined the impact of UVB or blue light treatment on health-promoting components in fruits, and less information is available for other lights on this subject.

Newhall navel orange (Citrus sinensis Osbeck) is one of the most important orange cultivars distributed worldwide for its special flavor and good performance during storage [18]. The fruits have high nutritional value, and are abundant in hesperidin, narirutin, and limonin. These compounds present health-promoting properties such as antioxidant, anticancer, anti-atherosclerotic, and vasodilatation effects $[19,20]$. Due to its high overall quality, Newhall navel orange has gradually become one of the leading fresh citrus cultivars in China [18,21]. Currently, China is the largest producers of Newhall navel orange in the world. By the end of 2018, the total planting area in China had reached around 2000 thousand hectares, with an annual output of approximately 4 million tons, and play an important role in citrus production in China. In this work, we investigated the effect of both UV and LED irradiation on individual flavonoids and limonoids in the edible portion (segments) of Newhall navel orange to determine the optimal light quality for maintaining or improving nutritional quality during storage.

\section{Results}

\subsection{Identification of Flavonoids and Limonoids}

From 2-16 min of retention time, every ion peak was analyzed in both low- and high-energy modes (Supplementary Materials Figures S1-S22). Each identified compound exhibited extremely similar retention times, accurate mass, and $\mathrm{MS}^{2}$ ion fragments with reported information for the same compound (Table 1 and Supplementary Materials Table S1) [19]. For example, in the first observed flavonoid, the ion peak was found at $3.36 \mathrm{~min}$ with the quasi-molecular ion $[\mathrm{M}+\mathrm{H}]^{+}$at $\mathrm{m} / z 595.1659$ in the low-energy mode (Figure 1a), corresponding to the reported retention time at $3.44 \mathrm{~min}$ with the MS ion at $\mathrm{m} / \mathrm{z} 595.1652$ (Table S1). Through elementary composition matching, the molecular formula was identified as $\mathrm{C}_{27} \mathrm{H}_{30} \mathrm{O}_{15}$. In addition, in the highenergy mode, prominent $\mathrm{MS}^{2}$ ion fragments were observed at $\mathrm{m} / \mathrm{z} 595.16565,325.07041,457.11183,379.08087$, 409.09118, and 477.11946 (Figure 1b), corresponding to the reported diagnostic ions at $\mathrm{m} / \mathrm{z} 595.16484[\mathrm{M}+\mathrm{H}]^{+}$,

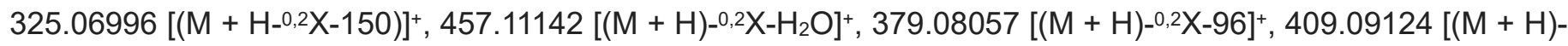
$\left.150-2 \cdot \mathrm{H}^{2} \mathrm{O}\right]^{+}$and $477.12020\left[{ }^{1,3} \mathrm{~A}^{+}\right]$(Supplementary Materials Table S1). Therefore, the first compound was identified as apigenin-6,8-di-C-glucoside (vicenin-2). In total, twenty-one health-promoting phytochemicals were identified in the segments of Newhall navel oranges, including six polymethoxylated flavonoids, five flavone-O/Cglycosides, seven flavanone-O-glycosides, and three limonoids (Table 1). 


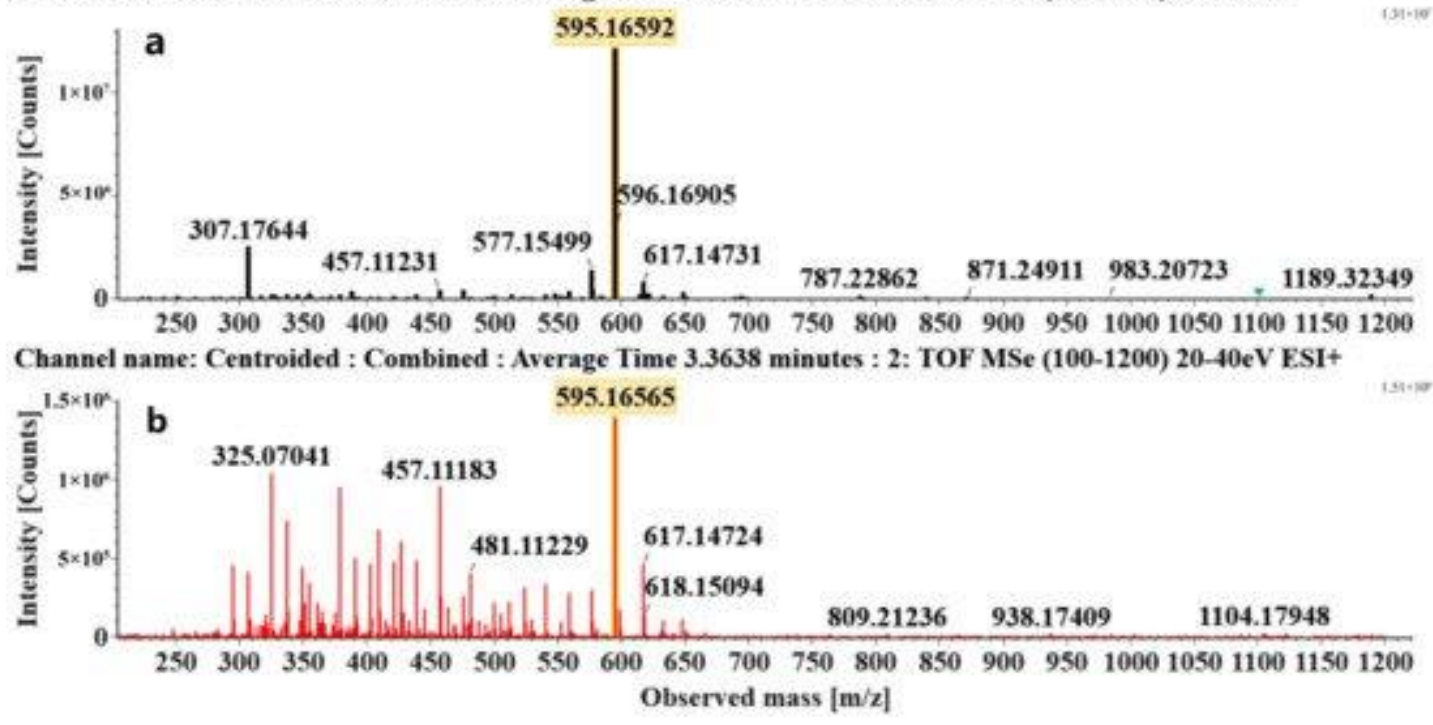

Figure 1. Mass chromatogram of apigenin-6,8-di-C-glucoside (Vicenin-2) in positive (a) low-energy and (b) highenergy modes.

Table 1. Summary of the 21 compounds identified.

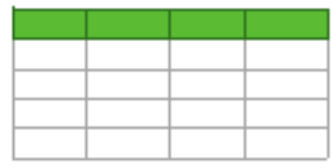

After identification, the peak area of $[\mathrm{M}+\mathrm{H}]^{+}$for each compound was integrally computed to acquire MS response values (Supplementary Materials Figures S23-S43), which were directly used to represent the content of each compound without further quantification.

\subsection{Effect of Light Irradiation on Flavonoids and Limonoids in the Segments of Newhall Navel Oranges}

Six PMFs, namely, 5,7,8,3',4'-pentamethoxyflavone (isosinensetin), 5,6,7,8,4'-pentamethoxyflavone

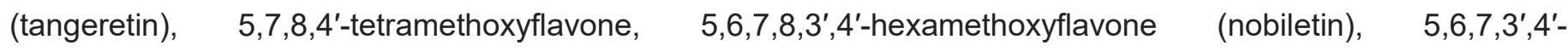
pentamethoxyflavone (sinensetin), and 3,5,6,7,8,3',4'-heptamethphoxyflavone, were identified from the segments of Newhall navel oranges. The accumulation of these identified PMFs was altered in response to LED and UV light irradiation (Figure 2). In spite of light irradiation, the contents of all PMFs identified in this study significantly decreased during the irradiation period, but red and blue light maintained higher levels of PMFs compared to other lights. After removal from light irradiation, the accumulation of PMFs continued to decrease in fruit treated with red and blue irradiation, and darkness, while their content was significantly induced by white light, UVB, and UVC irradiation, with white light irradiation exhibiting the optimal effect followed by UVB (Figure 2). 


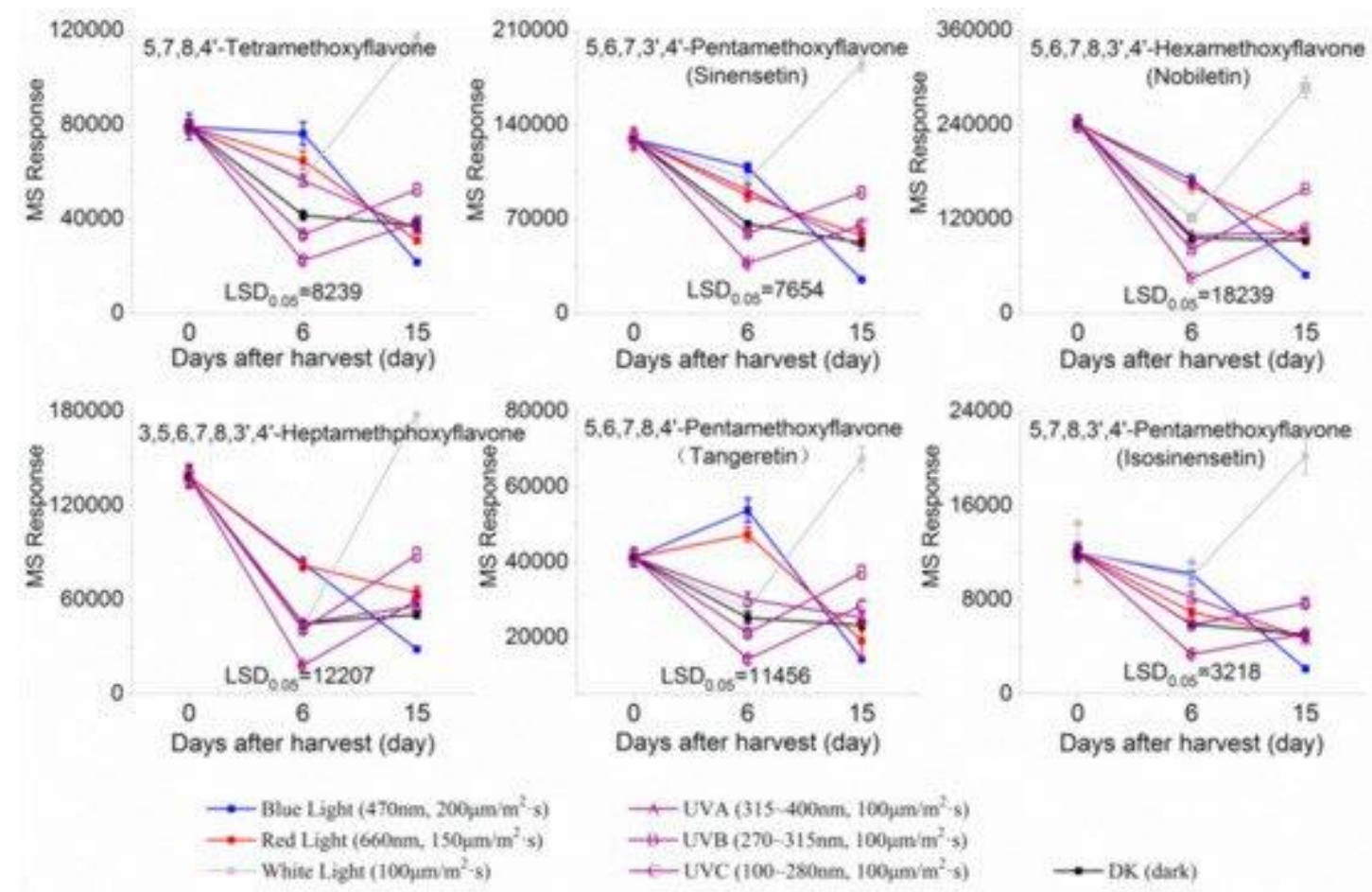

Figure 2. MS response of PMFs in the segments of Newhall navel orange during light irradiation and the removal period.

Twelve flavonoid glycosides were identified in the segments of Newhall navel oranges, specifically five flavone-O/C-glycosides (apigenin-6,8-di-C-glucoside, diosmetin-6,8-di-C-glucoside, rhoifolin-4'-O-glucoside, chysoeriol-6,8-di-C-glucoside, diosmin) and seven flavanone-O-glycosides (hesperidin, didymin, neoeriocitrin, narirutin, neoeriocitrin, narirutin-4'-glucoside, eriocitrin). Among these compounds, two flavone glycosides (diosmin, diosmetin 6,8-di-C-glucoside) and four flavanone glycosides (hesperidin, didymin, neoeriocitrin, narirutin) were increased in response to red light irradiation throughout the entire experimental period (Figure 3a). Similarly, narirutin, neoeriocitrin and didymin were also induced by UVA, UVB and UVC in spite of irradiation or removal from treatment. In the dark, the accumulation of diosmin, diosmetin 6,8-di-C-glucoside, hesperidin, didymin, and narirutin continued to decrease during the irradiation period, but consistently increased when the fruits were moved to natural light (Figure 3a). In contrast, the six flavonoids in Figure 3a were induced by blue light during the irradiation period, but their content rapidly decreased when removed from blue light, except for narirutin. In Figure 3b, UVA, UVB, UVC, darkness, and red light induced the accumulation of vicenin-2, rhoifolin4'-O-glucoside, and narirutin-4'-glucoside throughout the entire experimental period; UVC exhibited the greatest inducing effect, and white light irradiation promoted lower levels of these compounds. However, even blue light irradiation induced the accumulation of the four flavonoids, and this effect disappeared when blue light was removed (Figure $3 b$ ). In addition, eriocitrin and neohesperidin were significantly induced by red light and UVB throughout the entire experimental period, with UVC and white light resulting in remarkable decreases in two flavonoids during the irradiation period and inducing a sharp increase when these two lights were removed. Fruit irradiated with blue light exhibited an inverse response (Figure 3c). 

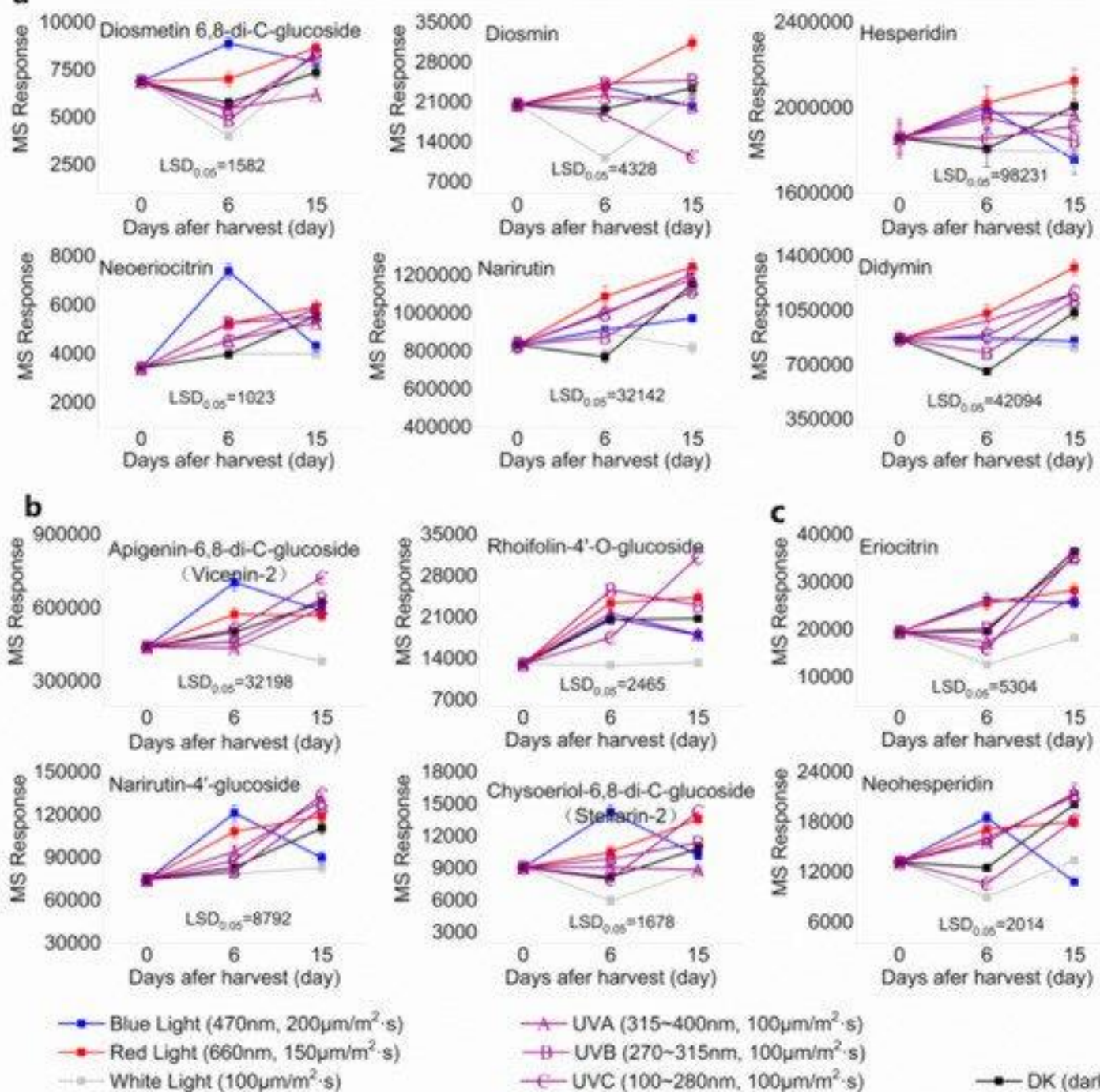

A-UVA $\left(315 \sim 400 \mathrm{~nm}, 100 \mu \mathrm{m} / \mathrm{m}^{2} \cdot \mathrm{s}\right)$

\#-UVB $\left(270-315 \mathrm{~nm}, 100 \mu \mathrm{m} / \mathrm{m}^{2} \cdot \mathrm{s}\right)$

- UVC $\left(100 \sim 280 \mathrm{~nm}, 100 \mu \mathrm{m} / \mathrm{m}^{2} \cdot \mathrm{s}\right)$

$\rightarrow$ DK (dark)

Figure 3. MS response of flavone and flavanone in the segments of Newhall navel orange during light irradiation and during the removal period. Flavone and flavanone (a) were mostly induced by red light, (b) UVC, and were also (c) induced by UV and darkness.

Three limonoids were identified, namely, limonin, epilimonin, and 7a-limonyl acetate, all of which exhibited patterns of increasing accumulation when the fruits were treated with UVA, UVB, UVC, and red and white light. In contrast, the content of limonin decreased when these forms of irradiation were removed, but the content of epilimonin in red light- and UVB-treated fruits and 7a-limonyl acetate in UVA- and UVB-treated fruits increased when irradiation was removed. Furthermore, the content of these three identified limonoids decreased during treatment with blue light and darkness but increased when irradiation was removed (Figure 4).

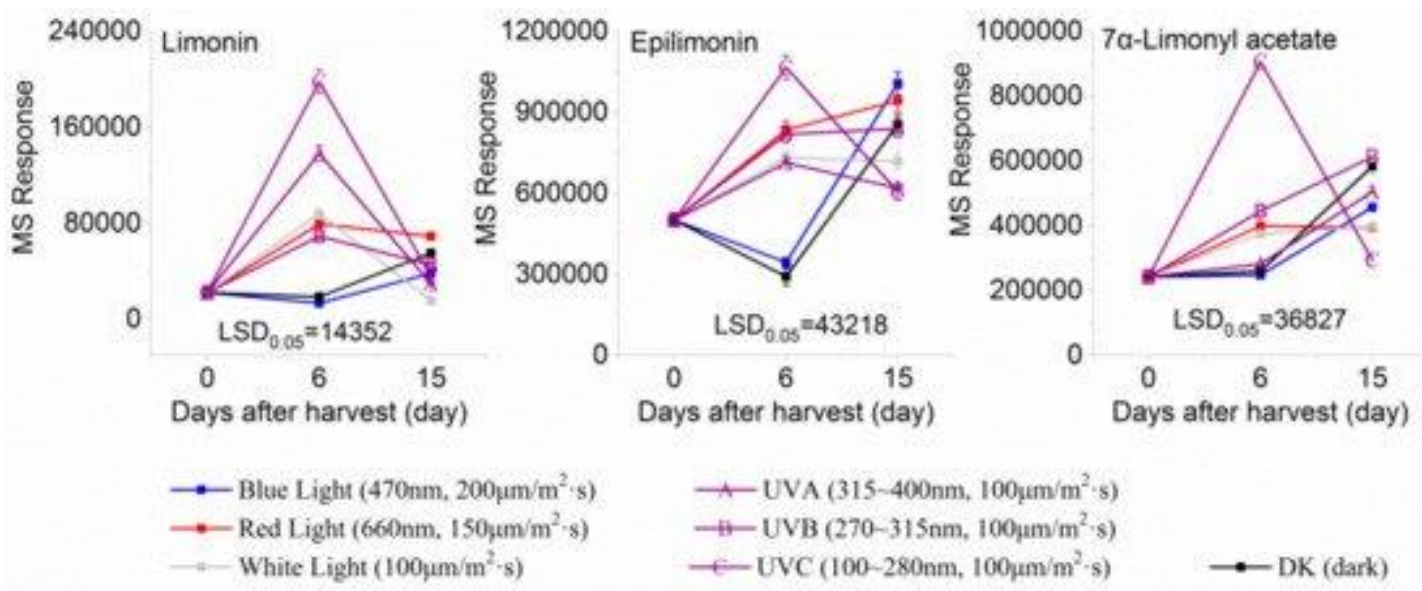

Figure 4. MS response of limonoids in the segments of Newhall navel orange during light irradiation and during the removal period. 


\subsection{Principal Component Analysis of Flavonoid and Limonoid Responses}

Principal component analysis (PCA) was performed based on the MS response value of 21 flavonoids and limonoids identified in response to different light treatments (UVC, UVB, UVC, red, blue, white light, and darkness) and storage times $(0,6,15$ days). As shown in Figure 5, the variance explained by PC1, PC2, and PC3 was 39.8, 29.1 , and $9.4 \%$, respectively. The identified compounds were clearly distinguished according to exposure to different sources of light irradiation. Polymethoxylated flavonoids (PMFs) were clustered with white light treatment sampled at storage day 15 (WL-15 day). Flavonoid glycosides were clustered with red and UVC light treatment sampled at storage day 15 (RL-15 day, UVC-15 day), and limonoids were clustered with UVC treatment at storage day 6 (UVC-6 day). Taken together, the results of the principal component analysis are in agreement with our observations of MS response.

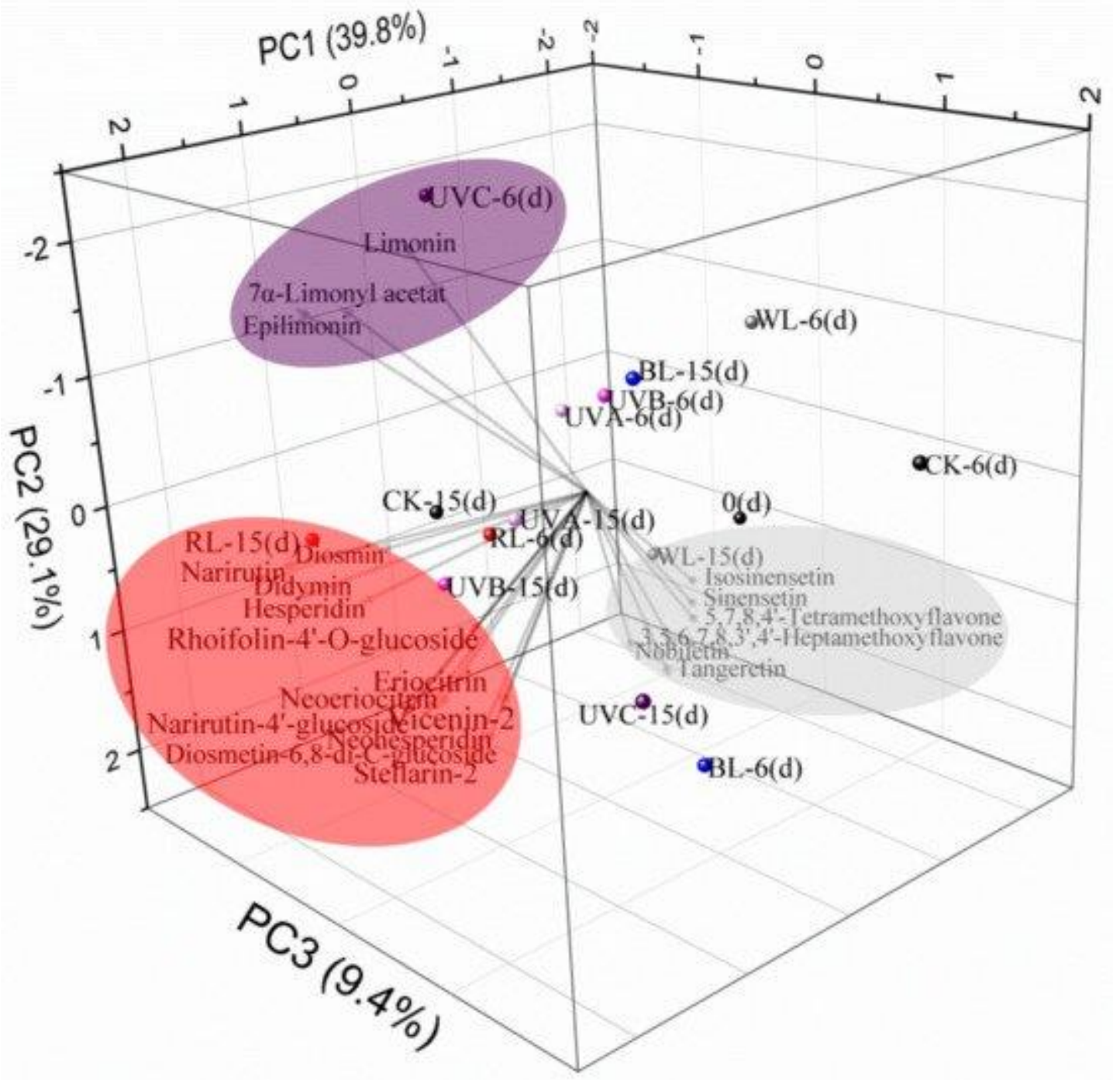

Figure 5. PCA model based on 21 phytochemicals in Newhall navel orange in response to different forms of light irradiation. DK: Dark; WL: White light; BL: Blue light; RL: Red light; UVA: Ultraviolet A; UVB: Ultraviolet B; UVC: Ultraviolet C; d: Days after harvest.

\section{Discussion}

Light irradiation has been developed as an efficient method with no pollution to extend the shelf-life and maintain the quality of vegetables and fruits. In general, visible and ultraviolet light are both intrinsic parts of sunlight, while in the natural environment, all solar UVC $(<280 \mathrm{~nm})$ and most UVB $(280-315 \mathrm{~nm})$ rays are absorbed by the stratospheric ozone layer before reaching the Earth's surface [37]. Artificial light, especially LED light that can be set to any wavelength, and is widely used in research on supplementary irradiation of various plant species. In recent years, the majority of studies on fruits treated with light irradiation have reported increases in total flavonoid content, but few studies have focused on the effect of light on individual flavonoids. For most studies, only one monochromatic light was used, while almost no studies have reported the effect of multiple light irradiation 
sources alone on flavonoids. In addition, all studies only investigated the effect of irradiation period. Few available studies concerned the delay effects when irradiation was removed. For citrus fruits, a relevant study reported that blue light (450 nm, $\left.630 \mu \mathrm{mol} / \mathrm{m}^{2} \cdot \mathrm{s}\right)$ induces some phenylpropanoids, such as scoparone [38], and, as reported previously, naringin and tangeretin are induced by UVC irradiation (254 nm, $\left.0.1 \mathrm{~W} / \mathrm{m}^{2}\right)$ [39]. In this study, we investigated the effect of post-harvest light irradiation on the accumulation of individual flavonoids and limonoids in the segments of Newhall navel orange simultaneously, using five kinds of LEDs and ultraviolet light within the wavelength range of $100-660 \mathrm{~nm}$, as well as one type of composite white light $\left(100 \mu \mathrm{m} / \mathrm{m}^{2} \cdot \mathrm{s}\right)$ and darkness, to identify the optimal light treatment for maintaining and improving health-promoting components in citrus fruits.

Herein, we detected 21 phytochemicals in the segments of Newhall navel oranges (Citrus sinensisOsbeck) and further identified six PMFs, twelve flavonoid glycosides, and three limonoids, which are almost identical to citrus characteristic compounds reported previously $[40,41,42]$. Previous studies demonstrated that red and blue light treatment increases flavonoid content in microgreens [43]. Blue light, rather than red light, enhances the accumulation of flavonoids in callus cultures of Saussurea [44]. In the present study, we found that even red and blue light could not induce the accumulation of PMFs in the segments of Newhall navel oranges. However, red and blue light maintained higher levels of PMFs compared to other light sources during the irradiation period. More importantly, post-harvest white light $\left(100 \mu \mathrm{m} / \mathrm{m}^{2} \cdot \mathrm{s}\right)$ irradiation specifically enhanced the accumulation of PMFs when light was removed, suggesting that each compound responds differently to a single light source. In Arabidopsis thaliana, key enzymes of anthocyanin biosynthesis are strongly induced by blue light, and the induction intensity decreases in the order of UVA, UVB, and red light [45]. In this study, despite the content of twelve flavonoid glycosides all obviously increasing during blue light irradiation, these contents were dramatically decreased after removal of the light. Unlike blue light, red light significantly increased the content of diosmetin6,8-di-C-glucoside, diosmin, hesperidin, neoeriocitrin, narirutin, and didymin. Furthermore, UVC remarkably elevated levels of vicenin-2, rhoifolin-4'-O-glucoside, narirutin-4'-glucoside, and stellarin-2 in fruit segments, and the accumulation effects remained after removal of the light source until the last day of observation, which was on day 15 of storage. Therefore, red light and UVC irradiation might represent a more efficient method for improving flavonoid content after harvest, which is an essential demand of post-harvest logistic systems of fresh fruits.

Compared to flavonoids, little information is available concerning the effect of light irradiation on the accumulation of limonoids. To our knowledge, the first relevant study was reported by Jairam et al. and demonstrated that limonin and nomilin were reduced in irradiated (300 gray) freeze-dried grapefruit pulp [46]. Our results show that only UVC irradiation induced limonin, epilimonin, and 7a-limonyl acetate during six days of continuous irradiation, despite the content decreasing again after removal of the light. Similar effects were observed in response to other light treatments. Although limonoids were first discovered as bitter citrus components, their rich biological activities were later found to promote human health, which is undoubtedly more important [40]. The above results demonstrated that both LED and UV light affect the metabolism of flavonoids and limonoids in citrus fruits.

A previous report found that irradiation of pear with UVB $(280-315 \mathrm{~nm})$ and/or fluorescent lamps for 10 days after harvest not only results in a significant increase in the anthocyanin, flavonoids, chlorophyll, and carotenoid concentration in the peel, but also leads to a slight increase in soluble solid and organic acid concentration and a significant increase in total sugars in the flesh [15], indicating that post-harvest irradiation can permeate through the peel of the whole fruit to affect the inner pulp. Our present study provides further evidence for this. Even so, besides the light quality, the intensity of light is another important factor affecting the accumulation of metabolites in fruits, especially in the inner part of the flesh. Therefore, when a specific intensity of light was used to irradiate fruit, the intensity of light may be different for each part of the fruit. For this point, further work is required to screen for the optional intensity of light for flesh.

Light-controlled flavonoid biosynthesis in fruits is known to be regulated by $R 2 R 3 M Y B$ transcription factors. Constitutive photomorphogenic 1 (COP1) acts as a central repressor in the light signaling pathway by interacting directly with photoreceptors to mediate flavonoid biosynthesis [47]. In visible light, the function of COP1 was repressed by interacting with activated photoreceptors, and COP1 is subsequently exported from the nucleus, allowing nuclear-localized transcription factors to accumulate and induce gene expression in light-regulated 
processes. The expression of structural flavonoid genes is directly regulated by $R 2 R 3 M Y B$ transcription factors which may be regulated by basic leucine zipper (bZIP) transcription factors such as HY5. Ultraviolet $\mathrm{B}$ radiation is strongly absorbed by tryptophan (Trp) amino acid residues in the dimeric form of a UV resistance locus 8 (UVR8) photoreceptor leading to the monomerization of UVR8. Monomeric UVR8 and COP1 form a complex that accumulates in the nucleus of the cells [48,49]. The UVR8-COP1-SPA complex stabilizes the bZIP transcription factor HY5, promoting the activity of different R2R3 MYBs for the transcription of specific flavonoid biosynthesis genes. However, the possible mechanism of limonoids in response to light irradiation needs to be further studied in the future.

\section{Materials and Methods}

\subsection{Chemicals}

The HPLC-grade acetonitrile and methanol were purchased from Sigma-Aldrich (St. Louis, MO, USA). The HPLC-grade formic acid was purchased from Fisher Scientific (Shanghai, China). Ultrapure water was acquired using the Millipore water purification System (Bedford, MA, USA).

\subsection{Sample Treatment}

Newhall navel oranges were collected in December 2018 from the National Citrus Germplasm Repository, Citrus Research Institute of Chinese Academy of Agricultural Sciences, Chongqing, China. Ten fruit trees with the same age and similar growth condition were marked at the same orchard before sampling. Fully ripe fruits were picked randomly from four directions on these marked trees. Then, the fruits were randomly divided into 7 groups, and 180 fruits were included in each group, and these were then treated with different wavelengths of light. For each sampling, 60 fruits were taken out, 20 fruits each as biological replicates. Treatment conditions were set using the RXZ-300D intelligent growth cabinet with adjustable light irradiation parameters, purchased from Southeast Instrument Company (Ningbo, China), and all the UV TL-D36/16 lamps were purchased from Philips Company (Netherland), and the LED RDN-500B-C lamps were purchased from Ningbo Southeast Instrument Co., Ltd. (Ningbo, China). The control group was stored at $20{ }^{\circ} \mathrm{C}$ and $90-95 \%$ relative humidity $(\mathrm{RH})$ in the dark. The other groups were irradiated with UVC (10-280 nm, $\left.100 \mu \mathrm{m} / \mathrm{m}^{2} \cdot \mathrm{s}\right)$, UVB (270-315 nm, $\left.100 \mu \mathrm{m} / \mathrm{m}^{2} \cdot \mathrm{s}\right)$, UVA (315$\left.400 \mathrm{~nm}, 100 \mu \mathrm{m} / \mathrm{m}^{2} \cdot \mathrm{s}\right)$, blue light $\left(470 \mathrm{~nm}, 200 \mu \mathrm{m} / \mathrm{m}^{2} \cdot \mathrm{s}\right)$, red light $\left(660 \mathrm{~nm}, 150 \mu \mathrm{m} / \mathrm{m}^{2} \cdot \mathrm{s}\right)$, and white light $(100$ $\mu \mathrm{m} / \mathrm{m}^{2} \cdot \mathrm{s}$ ) at $20{ }^{\circ} \mathrm{C}$ and $90-95 \%$ relative humidity $(\mathrm{RH})$ for 6 days. On day 6 , all lights were removed, while the same temperature and relative humidity conditions were maintained for 10 days. Fruits from each group were sampled at 0,6, and 15 days. Sample fruits were peeled to retain only the edible portion (segments) and were immediately frozen in liquid nitrogen, powdered, and stored at $-80^{\circ} \mathrm{C}$ until analysis.

\subsection{Extraction of Flavonoids and Limonoids}

Flavonoids and limonoids were crude extracted by methanol according to the method of Xing et al. [50] with slight modifications. Four grams of segment powder from oranges were fully mixed with $7 \mathrm{~mL}$ methanol. At room temperature, mixtures were placed in a 300-W ultrasonic bath for $30 \mathrm{~min}$ and centrifuged at $5000 \mathrm{rpm}$ for $20 \mathrm{~min}$. The supernatant was recovered in a $25-\mathrm{mL}$ brown volumetric flask. The precipitate was again extracted twice using the same method. All $21-\mathrm{mL}$ mixed supernatants were finally diluted using methanol to $25 \mathrm{~mL}$ in brown volumetric flasks. Methanol extracts were filtered using 13-mm syringe filters with 0.22- $\mu \mathrm{m}$ PTFE membranes before passing through the UPLC column.

\subsection{UPLC-qTOF-MS Analysis and MS Response Quantification}

Samples were analyzed using UPLC/Xevo G2-S Q-TOF MS (Waters MS Technologies, Manchester, U.K.), equipped with an ACQUITY UPLC BEH C18 column $(2.1 \times 100 \mathrm{~mm}, 1.7 \mathrm{~mm}$, UK) coupled with the UNIFI Platform in triplicate. The analysis method was performed according to Zhao et al. [51]. Briefly, the column heater was set at $40{ }^{\circ} \mathrm{C}$ and $1 \mu \mathrm{L}$ of each sample was injected and eluted with $0.1 \%$ formic acid in water (solvent $\mathrm{A}$ ) and acetonitrile (solvent B) with a linear gradient (0-3 min, 5-15\% B; 3-8 min, 15-25\% B; 8-9 min, 25-35\% B; 9-13 min, 35-45\% B; $13-15 \mathrm{~min}, 45-60 \% \mathrm{~B} ; 15-16 \mathrm{~min}, 60-90 \% \mathrm{~B} ; 16-18 \mathrm{~min}, 90-90 \% \mathrm{~B} ; 18-20 \mathrm{~min}, 90-5 \% \mathrm{~B}$ ) at a flow rate of $0.4 \mathrm{~mL} / \mathrm{min}$. Mass spectrometry was performed in the positive ionization mode in both low- $(6 \mathrm{~V})$ and high-energy $(20-40 \mathrm{~V})$ conditions. Mass spectrum conditions were as follows: source temperature of $120{ }^{\circ} \mathrm{C}$, desolvation temperature of $400{ }^{\circ} \mathrm{C}$, capillary voltage of $1000 \mathrm{~V}$, cone voltage of $40 \mathrm{~V}$, cone gas flow of $50 \mathrm{~L} / \mathrm{h}$ and desolvation 
gas flow rate of $800 \mathrm{~L} / \mathrm{h}$. Leucine-enkephalin $(\mathrm{m} / \mathrm{z556.2766)}$ was used as the lock-mass compound at a concentration of $200 \mathrm{ng} / \mathrm{mL}$ and flow rate of $10 \mu \mathrm{L} / \mathrm{min}$. Mass spectrometry data were collected between $\mathrm{m} / \mathrm{z} 100$ and 1200. The identification and quantification of target compounds were performed according to the previous methods [50,51]. The target compounds were identified by the retention time, quasi-molecular ion, molecular formula, and dominant or diagnostic $\mathrm{MS}^{2}$ ion, and MS responses represent the relative content of the compounds.

\subsection{Statistical Analysis}

The peak area of the quasi-molecular ion for each identified compound was integrally computed using UNIFI software (Waters, Milford, MA, USA). Statistical differences of compound contents for each treatment point were evaluated by Fisher's protected least squares difference (LSD) test at a 0.05 probability. Data were expressed as the mean of three biological replicates \pm standard deviation (SD), using IBM SPSS Statistics software v.23 for analyses. To determine all the variables attributed to differentiate different samples, a PCA model was developed using the contents of all target compounds as the independent variables and the treatment times as the dependent variables, respectively. Three line charts and principal component analysis (PCA) were plotted by Origin Pro 2018 (Origin Lab, Northampton, MA, USA).

\section{Conclusions}

Twenty-one flavonoids were identified in the segments of Newhall navel oranges, specifically six PMFs, five flavone glycosides, seven flavanone glycosides, and three limonoids. The accumulation of these compounds was altered in response to exposure to different LED and UV light irradiation. The levels of PMFs decreased significantly during the irradiation period, and red and blue light maintained higher levels of PMFs. White light and UVB significantly induced the accumulation of PMFs after the fruits were removed from irradiation. Red light enhanced the content of diosmetin 6,8-di-C-glucoside, diosmin, hesperidin, neoeriocitrin, narirutin, and didymin throughout the entire experimental period with light irradiation, whereas UVC enhanced the accumulation of vicenin-2, rhoifolin-4'-O-glucoside, narirutin-4'-glucoside, and stellarin-2. Three limonoids, limonin, epilimonin, and 7a-limonyl acetate, were induced by UVC during the irradiation period but decreased when the irradiation was removed. These results suggest that single light sources, such as red light and UVC, have great potential for improving the nutrition and flavor of citrus fruits, which can be used for post-harvest logistic systems and lighting conditions for supermarket sales. However, the specific molecular mechanisms underlying these effects require further investigation.

\section{References}

1. Chen, S. A.; Chang, E. C.; Chuang, K. R.; Chao, C. I.; Wei, P. K.; Fann, W. S., Conjugated polymer blends as emitting layer for white light led. Abstr Pap Am Chem S 1998, 215, U392-U392.

2. Goustouridis, D.; Manoli, K.; Chatzandroulis, S.; Sanopoulou, M.; Raptis, I., Characterization of polymer layers for silicon micromachined bilayer chemical sensors using white light interferometry. Sensor Actuat B-Chem 2005, 111, 549554.

3. Chuang, C. Y.; Shih, P. I.; Chien, C. H.; Wu, F. I.; Shu, C. F., Bright-white light-emitting devices based on a single polymer exhibiting simultaneous blue, green, and red emissions. Macromolecules 2007, 40, 247-252.

4. Chitara, B.; Bhat, S. V.; Vivekchand, S. R. C.; Gomathi, A.; Rao, C. N. R., White-light sources based on composites of gan nanocrystals with conducting polymers and nanophosphors. Solid State Commun 2008, 147, 409-413.

5. Cheng, G.; Fei, T.; Zhao, Y.; Ma, Y. G.; Liu, S. Y., White phosphorescent polymer light-emitting devices based on a wide band-gap polymer derived from 3,6-carbazole and tetraphenylsilane. Org Electron 2010, 11, 498-502.

6. Amin, G.; Zaman, S.; Zainelabdin, A.; Nur, O.; Willander, M., Zno nanorods-polymer hybrid white light emitting diode grown on a disposable paper substrate. Phys Status Solidi-R 2011, 5, 71-73. 
7. Chang, C. J.; Lai, C. F.; Reddy, P. M.; Chen, Y. L.; Chiou, W. Y.; Chang, S. J., Color optimization of conjugatedpolymer/ingan hybrid white light emitting diodes by incomplete energy transfer. $J$ Lumin 2015, 160, 145-150.

8. Chen, B.; Feng, J. C., White-light-emitting polymer composite film based on carbon dots and lanthanide complexes. J Phys Chem C 2015, 119, 7865-7872.

9. Feng, C.; Sun, J. W.; Yan, P. F.; Li, Y. X.; Liu, T. Q.; Sun, Q. Y.; Li, G. M., Color-tunable and white-light emission of one-dimensional I-di-2-thenoyltartaric acid mixed-lanthanide coordination polymers. Dalton T 2015, 44, 4640-4647.

10. Biria, S.; Judge, K.; Malley, P.; Kahan, T.; Hosein, I., Controlling polymer morphology using white-light. Abstr Pap Am Chem S 2016, 252.

11. da Silva, M. A. T.; Thomazini, E. F.; Albertini, M.; Renzi, W.; Franchello, F.; Dias, I. F. L.; Duarte, J. L.; Pocas, L. C.; Lourenco, S. A., Characterization of digital textile printing and polymer blend (pfo-dmp:P3ht) for application in manufacture of organic diodes emitting white light - woleds. Opt Mater 2016, 62, 119-131.

12. Fan, L. M.; Fan, W. L.; Li, B.; Zhao, X.; Zhang, X. T., W-shaped 1,3-di(2,4-dicarboxyphenyl)benzene based lanthanide coordination polymers with tunable white light emission. New J Chem 2016, 40, 10440-10446.

13. Chen, M. X.; Sun, R. Y.; Ye, Y. C.; Tang, H. J.; Dong, X. Y.; Yan, J. L.; Wang, K. M.; Zhou, Q.; Wang, Z. L., Application of a novel red-emitting cationic iridium(iii) coordination polymer in warm white light-emitting diodes. Opt Mater 2018, 76, $141-146$.

14. Huang, J. S.; Li, G.; Wu, E.; Xu, Q. F.; Yang, Y., Achieving high-efficiency polymer white-light-emitting devices. Adv Mater 2006, 18, 114-117.

15. Kassamakov, I.; Ojala, K.; Salmia, A.; Haeggstrom, E.; Aaltonen, J.; Huber, A.; Saarikko, H.; Osterberg, M.; Oinonen, M., Characterization of dents and grooves on polymer films using scanning white light interferometry. Optical Micro- and Nanometrology in Microsystems Technology 2006, 6188.

16. Kim, T. H.; Lee, H. K.; Park, O. O.; Chin, B. D.; Lee, S. H.; Kim, J. K., White-light-emitting diodes based on iridium complexes via efficient energy transfer from a conjugated polymer. Adv Funct Mater 2006, 16, 611-617.

17. Nicolai, H. T.; Hof, A. J.; Blom, P. W. M., Charge transport in white light-emitting polymers. Organic Optoelectronics and Photonics li 2006, 6192.

18. Zhen, H. Y.; Xu, W.; King, W.; Chen, Q. L.; Xu, Y. H.; Jiang, J. X.; Peng, J. B.; Cao, Y., White-light emission from a single polymer with singlet and triplet chromophores on the backbone. Macromol Rapid Comm 2006, 27, 2095-2100.

19. Lee, P. I.; Hsu, S. L. C.; Lee, R. F., White-light-emitting diodes from single polymer systems based on polyfluorene copolymers end-capped with a dye. Polymer 2007, 48, 110-115.

20. Liedtke, A.; O'Neill, M.; Wertmoller, A.; Kitney, S. P.; Kelly, S. M., White-light oleds using liquid crystal polymer networks. Chem Mater 2008, 20, 3579-3586.

21. Inoue, A.; Hosokawa, T.; Haishi, M.; Ohtani, N., 4-(dicyanomethylene)-2-methyl-6-(p-dimethylaminostyryl)-4h-pyran $(\mathrm{dcm})$-doping density dependence of luminescence spectra and white emission in polymer light-emitting diodes. Physica Status Solidi C - Current Topics in Solid State Physics, Vol 6, No 1 2009, 6, 334-337. 
22. Jeon, S. O.; Joo, C. W.; Yook, K.; Lee, J. Y., Color control of multilayer stacked white polymer light-emitting diodes using a quantum dot as an interlayer. Appl Phys Lett 2009, 94.

23. Wang, R.; Peng, J.; Qiu, F.; Yang, Y. L.; Xie, Z. Y., Simultaneous blue, green, and red emission from diblock copolymer micellar films: A new approach to white-light emission. Chem Commun 2009, 6723-6725.

24. Kim, S. H.; Jin, Y.; Yu, J. Y.; Kim, J.; Song, S.; Suh, H.; Lee, K., Color stable white polymer light-emitting diodes with single emission layer. Synthetic Met 2010, 160, 835-838.

25. Lee, P. I.; Hsu, S. L. C.; Lin, P. Y., White-light-emitting diodes from single polymer systems based on polyfluorene copolymers with quinoxaline derivatives. Macromolecules 2010, 43, 8051-8057.

26. Madhwal, D.; Rait, S. S.; Kumar, A.; Verma, A.; Tada, K.; Onoda, M.; Bhatnagar, P. K.; Mathur, P. C., Development and characterization of an efficient bio-white polymer light-emitting diode with red and green phosphorescent dyes as dopants. J Mater Sci 2010, 45, 3300-3303.

27. Hu, B.; Yao, C.; Huang, X. R., Designing of the white-light emission from a single-polymer system: Quantum theoretical study. Polym Sci Ser a+ 2011, 53, 1097-1105.

28. Lee, H. K.; Kim, T. H.; Park, J. H.; Kim, J. K.; Park, O. O., White-light-emitting diodes using miscible polymer blend doped with phosphorescent dye. Org Electron 2011, 12, 891-896.

29. Wang, R.; Peng, J.; Qu, F.; Yang, Y. L., Enhanced white-light emission from multiple fluorophores encapsulated in a single layer of diblock copolymer micelles. Chem Commun 2011, 47, 2787-2789

30. Jeon, Y. P.; Choo, D. C.; Kim, T. W., Color tunable white organic light-emitting devices with a hybrid 2-methyl-9,10di(2-naphthyl)anthracene small molecule/poly(2-methoxy-5-(2-ethyhexoxy)-1,4-phenylenevinylene) polymer emitting layer. Thin Solid Films 2012, 521, 189-192.

31. Meng, L. C.; Lou, Z. D.; Yang, S. Y.; Hou, Y. B.; Teng, F.; Liu, X. J.; Li, Y. B., White organic light-emitting diodes based on a combined electromer and monomer emission in doubly-doped polymers. Chinese Phys B 2012, 21.

32. Nicolai, H. T.; Hof, A.; Blom, P. W. M., Device physics of white polymer light-emitting diodes. Adv Funct Mater 2012, 22, 2040-2047.

33. Kim, J. H.; Song, W. S.; Yang, H., Color-converting bilayered composite plate of quantum-dot-polymer for high-color rendering white light-emitting diode. Opt Lett 2013, 38, 2885-2888.

34. Kuo, C. P.; Chuang, C. N.; Chang, C. L.; Leung, M. K.; Lian, H. Y.; Wu, K. C. W., White-light electrofluorescence switching from electrochemically convertible yellow and blue fluorescent conjugated polymers. J Mater Chem C 2013, $1,2121-2130$.

35. Liu, B. X.; Liu, Q. Q.; Tong, C. Y.; Lu, X. D.; Lu, C. L., Blue-light-emitting surface-functionalized zns nanoparticles and their transparent polymer nanocomposites with near-white light emission. Colloid Surface A 2013, 434, 213-219.

36. Leong-Hoi, A.; Claveau, R.; Flury, M.; Uhring, W.; Serio, B.; Anstotz, F.; Montgomery, P. C., Detection of defects in a transparent polymer with high resolution tomography using white light scanning interferometry and noise reduction. Proc Spie 2015, 9528. 
37. Nam, G. H.; Park, I. K., Cdse quantum dot-conducting polymer hybrid structure for phosphor-free white light-emitting diodes. J Korean Phys Soc 2015, 66, 785-789.

38. Kim, Y. J.; Park, B. C.; Park, J.; Kim, H. D.; Kim, N. H.; Suh, Y. D.; Kim, Y. K., White-light-emitting magnetite nanoparticle-polymer composites: Photonic reactions of magnetic multi-granule nanoclusters as photothermal agents. Nanoscale 2016, 8, 17136-17140.

39. Niu, W. Y.; Sun, J. W.; Yan, P. F.; Li, Y. X.; An, G. H.; Li, G. M., 2d I-di-toluoyl-tartaric acid lanthanide coordination polymers: Toward single-component white-light and nir luminescent materials. Chem-Asian J 2016, 11, 555-560.

40. Hrma, M.; Sichova, K.; Svoboda, J.; Vohlidal, J., Assembling of bis(tpy)fluorenes with zn2+ and fe2+ ions into metallo-supramolecular polymers with highly efficient white-light emission. Polymer 2017, 122, 22-33.

41. Lee, J. S.; Kang, B. H.; Kim, S. H.; Lee, J. W.; Lee, S. W.; Kim, S. W.; Gopalan, S. A.; Kwon, J. B.; Bae, J. H.; Kim, E. S.; Kwon, D. H.; Kang, S. W., All-solution-processed high-brightness hybrid white quantum-dot light-emitting devices utilizing polymer modified quantum dots. Org Electron 2017, 42, 393-398.

42. Li, B. N.; Liu, L.; Fu, G. R.; Zhang, Z.; Li, H. Y.; Lu, X.; Wong, W. K.; Jones, R. A., Color-tunable to direct white-light and application for white polymer light emitting diode (wpled) of organo-eu3+- and organo-tb3+-doping polymer. J Lumin 2017, 192, 1089-1095.

43. Shen, F. Z.; He, F.; Lu, D.; Xie, Z. Q.; Xie, W. J.; Ma, Y. G.; Hu, B., Bright and colour stable white polymer lightemitting diodes. Semicond Sci Tech 2006, 21, L16-L19.

44. Niu, Y. H.; Liu, M. S.; Ka, J. W.; Bardeker, J.; Zin, M. T.; Schofield, R.; Chi, Y.; Jen, A. K. Y., Crosslinkable holetransport layer on conducting polymer for high-efficiency white polymer light-emitting diodes. Adv Mater 2007, 19, 300+ .

45. Park, J. J.; Park, T. J.; Jeon, W. S.; Kim, S. Y.; Lee, Y. K.; Jang, J.; Kwon, J. H., White polymeric light-emitting diodes based on doping of an orange ir complex in a fluorene blue polymer. Idw '07: Proceedings of the 14th International Display Workshops, Vols 1-3 2007, 1033-1036.

46. Tang, K. C.; Tseng, S. R.; Li, W. S.; Meng, H. F.; Horng, S. F.; Hsu, C. S., Broad band and white phosphorescent polymer light-emitting diodes in multilayer structure. Synthetic Met 2008, 158, 287-291.

47. Park, J. J.; Park, T. J.; Jeon, W. S.; Kim, S. Y.; Lee, Y. K.; Jang, J.; Kwon, J. H., White polymeric light-emitting diodes based on doping of an orange ir complex in a fluorene blue polymer host. Mol Cryst Liq Cryst 2009, 498, 290-297.

48. Prakash, A.; Katiyar, M., White polymer light emitting diode using blend of fluorescent polymers. 16th International Workshop on Physics of Semiconductor Devices 2012, 8549.

49. Qin, L. J.; Zhu, Y. C.; Yang, H.; Ding, L.; Sun, F.; Shi, M.; Yang, S. P., White-light phosphorescence from binary coordination polymer nanoparticles. Mater Chem Phys 2013, 139, 345-349.

50. Song, H. J.; Shin, G. J.; Choi, K. H.; Lee, S.; Moon, D. K., White polymer light emitting diode materials introducing dendritic quinoxaline derivative: Synthesis, optical and electroluminescent properties. Synthetic Met 2014, 190, 1-7.

51. Sun, C.; Zhang, Y.; Sun, K.; Reckmeier, C.; Zhang, T. Q.; Zhang, X. Y.; Zhao, J.; Wu, C. F.; Yu, W. W.; Rogach, A. 
L., Combination of carbon dot and polymer dot phosphors for white light-emitting diodes. Nanoscale 2015, 7, 1204512050. 